\title{
Evaluación de bienestar de vacas lecheras en sistema de producción a pequeña escala aplicando el protocolo propuesto por Welfare Quality ${ }^{\circledR}$
}

\author{
Evaluating dairy cow wellbeing in small-scale production \\ systems with the Welfare Quality ${ }^{\circledR}$ protocol
}

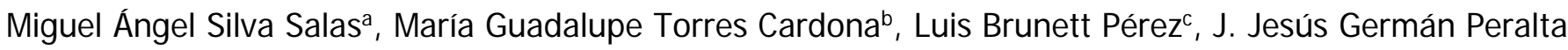 \\ Ortizb ${ }^{b}$ María del Rosario J iménez-Badillo ${ }^{a^{*}}$
}

\begin{abstract}
RESUMEN
La información de bienestar animal (BA) en las unidades de producción (UP) de México es escasa, por lo que el objetivo de este estudio fue evaluar el grado de bienestar de vacas lecheras en producción, mediante la aplicación del protocolo europeo Welfare Quality $^{\circledR}\left(W Q^{\circledR}\right)$ en cuatro municipios del estado de Hidalgo. Se evaluaron 1,187 vacas en producción, secas y vaquillas gestantes, distribuidas en 52 UP, seleccionadas por pertenecer al sistema familiar y participación voluntaria. En los formatos de $W Q^{\circledR}$ se recabó la información por encuesta estructurada, medición de recursos y a partir de la observación animal, con ajustes a las condiciones del sistema de producción y respetando el orden y tiempo sugerido por el protocolo. En el análisis de la información se aplicaron los algoritmos de árbol de decisiones, suma ponderada y funciones I-Spline y la integral de Choquet. La clasificación final coloca a todas las UP como aceptables y corroborado en el simulador de $W Q^{\circledR}$ en línea. La calificación en cada Principio (P) fue de 39 puntos para P1, 48 para P2, 23 en P3 y P4 con 28 puntos. Aun cuando el protocolo es una herramienta útil para la valoración del bienestar animal en el sistema de producción a pequeña escala del altiplano mexicano, la aplicabilidad no es total, por ello se hace necesario el desarrollo de protocolos propios, validados y probados científicamente que representen más fehacientemente la realidad del bienestar animal en este sistema.
\end{abstract}

PALABRAS CLAVE: Vacas lecheras, Bienestar animal, Welfare Quality ${ }^{\circledR}$, Pequeña escala.

\begin{abstract}
Animal welfare data from production units in Mexico is scarce. An evaluation was done of degree of wellbeing in producing dainy cows by applying the European Welfare Quality ${ }^{\circledR}\left(W^{\circledR}\right)$ protocol in four municipalities in the state of Hidalgo, Mexico. A total of 1,187 cows were evaluated in producing, dry or pregnant stages. Fifty-two (52) production units were chosen for being smallscale family systems and because producers voluntarily chose to participate. Data collection for the WQ ${ }^{\circledR}$ formats was by structured survey, resource measurement and animal observation, adjusted to the evaluated production systems and following the order and times suggested in the protocol. Four algorithms were used in data analysis: decision tree; weighted sum; i-spline functions; and Choquet integrals. All the evaluated production units were classified as acceptable, which was corroborated in the WQ ${ }^{\circledR}$ online simulator. The scores for each Principle (P) were 39 points for P1, 48 for P2, 23 for P3, and 28 for P4. This protocol is clearly a useful tool for evaluating animal wellbeing in small-scale production systems in the high plains of Mexico. However, it is not completely applicable to these systems. Validated, scientifically-tested protocols are needed that more reliably represent the reality of animal wellbeing in these systems.
\end{abstract}

KEY WORDS: Dairy cows, Animal wellbeing, Welfare Quality ${ }^{\circledR}$, Small-scale.

\footnotetext{
Recibido el 12 de octubre de 2015. Aceptado el 13 de enero de 2016.

a Centro Universitario UAEM Amecameca, Universidad Autónoma del Estado de México, México.

b Área Académica de Medicina Veterinaria y Zootecnia, Instituto de Ciencias Agropecuarias, Universidad Autónoma del Estado de Hidalgo, México.

c Instituto de Ciencias Agropecuarias y Rurales, Universidad Autónoma del Estado de México, México.

* Autor de correspondencia: mdjimenezb@uaemex.mx.

Proyecto financiado por Fundación Hidalgo Produce A.C.
} 


\section{INTRODUCCIÓN}

Bienestar animal (BA) es el modo en que un animal afronta las condiciones en las que vive; su origen es multifactorial, por lo tanto no se puede medir a partir de un solo indicador y no se infiere a partir de parámetros externos ${ }^{(1)}$; en esta tarea se debe aplicar una amplia variedad de indicadores fiables, validados y medibles que converjan en un orden de aplicación y análisis(2), donde la etología toma un papel importante ${ }^{(3)}$ para enfocar el bienestar a partir del animal y no de inducciones antropomorfistas $^{(4)}$. Mejorar el BA requiere la identificación de forma fiable de los aspectos que lo deterioran a fin de priorizar las acciones correctivas ${ }^{(5)}$, tomando en cuenta las condiciones particulares y aquellos aspectos socioculturales en los cuales se encuentre inmerso el sistema de producción.

Mediante pruebas científicas se determina el grado de bienestar del animal en relación a si está sano, cómodo, bien alimentado, en seguridad, capaz de expresar comportamiento innato, y si no padece sensaciones desagradables como el dolor, el miedo y la angustia(6).

Los criterios de evaluación del bienestar animal han evolucionado a partir de diferentes indicadores hasta llegar a una visión holística. El protocolo Welfare Quality ${ }^{\circledR}\left(W Q^{\circledR}\right)$ de la Unión Europea, se generó con la intención de integrar el BA en la cadena de producción de los alimentos como una estrategia para el desarrollo de políticas de producción de alimentos sustentables que permiten al consumidor elegir entre productos con valor diferenciado ${ }^{(3,7)}$.

Dicho protocolo elaborado por expertos en distintas disciplinas contempla todos los factores que intervienen en la producción: instalaciones, nutrición, sanidad, manejo zootécnico y relación humanoanimal(8); ha sido elaborado bajo condiciones de producción europea y se ha demostrado su aplicabilidad en diversas investigaciones como: calidad del bienestar en vacas lecheras en sistemas pastoriles en Brasil(9), valoración del BA de vacas lecheras en granjas comerciales en Argelia y Chile ${ }^{(10,11)}$, abordaje epidemiológico a los problemas más importantes en las granjas lecheras en Francia ${ }^{(5)}$, identificación de los desórdenes de salud más comunes en hatos lecheros ${ }^{(12)}$ y evaluar sistemas de alojamiento y su efecto en el $\mathrm{BA}^{(13)}$.

El sistema de producción a pequeña escala en México tiene sus propias implicaciones con respecto al BA; se ubica en la región templada del altiplano y aunque aporta solo el $10 \%$ de la producción nacional|(14), su importancia radica en la gran cantidad de pequeños productores que subsisten de éste, caracterizado por su número reducido de vacas $(<100)$ tipo Holstein en su mayoría, producción promedio por lactancia 4,800 L, tecnología media o baja ${ }^{(15)}$, brinda autoempleo y arraigo.

\section{MATERIAL Y MÉTODOS}

\section{Unidades de producción (UP)}

La presente investigación se realizó en los municipios de Tulancingo, Metepec, Acatlán y Cuautepec, en el Estado de Hidalgo, México. Durante octubre a diciembre de 2013 se realizaron reuniones de trabajo con las autoridades municipales y las agrupaciones de productores, a fin de contactar a los productores y obtener la localización física de las UP para elaborar la logística de visita y seleccionar las UP participantes con base a dos criterios: que su sistema de producción se clasificara en familiar o pequeña escala (con un número menor a 100 vacas), y que quisieran participar de forma voluntaria en este estudio.

De acuerdo a las normas establecidas para seleccionar las UP en este estudio y la flexibilidad del protocolo $W Q^{\circledR}$ para evaluar el total de las vacas por unidad de producción, éste se aplicó a 1,187 vacas (en producción, secas y vaquillas gestantes) de 52 unidades de producción con promedio de $22.8 \pm$ 15.0 vacas.

\section{Recolección de datos}

Para recabar la información se utilizaron los formatos sugeridos por el $W Q^{\circledR}$, que incluyen 4 principios, 12 criterios y 30 medidas (29 para este estudio) y fueron adaptados, probados y validados en campo en una muestra de 10 UP previo al trabajo final; su viabilidad, fiabilidad y repetibilidad en condiciones europeas se establecieron en estudios previos $^{(2)}$. 
Tres evaluadores con experiencia en vacas lecheras y entrenados en la utilización del protocolo $W Q^{\circledR}$, realizaron una visita única a cada UP de enero a marzo de 2014; cada uno de ellos realizó siempre la valoración de la misma medida en un orden establecido; el tiempo empleado fue el sugerido por el protocolo para cada una de las medidas, con la finalidad de interferir lo menos posible en el comportamiento normal de las vacas; estas actividades se realizaron después del ordeño matutino, con una duración variable de acuerdo al tamaño del hato.

La evaluación al ser de carácter no invasivo, se obtuvo de tres formas:

a) Observación de las vacas y asignación de medida en estática y dinámica:

En estática: condición corporal mediante la técnica de Edmonson ${ }^{(16)}$ y asignando un valor de 0 a vacas con condición normal, 1 muy flacas y 2 muy gordas, de acuerdo a lo establecido por el protocolo $W Q^{\circledR}$; nivel de limpieza de patas, ubres y flancos con la técnica de Hughes ${ }^{(17)}$; presencia de alteraciones tegumentarias, secreción nasal, ocular, vulvar y diarrea ${ }^{(12,18)}$

En dinámica en las áreas de pastoreo: valoración de cojeras, abordaje o zona de fuga ${ }^{(19,20)}$; comportamiento y tiempo requerido para echarse $^{(18)}$.

b) Revisión y medición de instalaciones (datos de los recursos) que incluye la limpieza, funcionamiento y tamaño de bebederos/abrevaderos, tanto en la unidad de producción como en la zona de pastoreo.

c) Entrevista al productor o encargado del hato (datos a partir de procesos) a través de una encuesta estructurada para determinar tiempo de pastoreo, uso o no de analgésicos y anestésicos en descorne, y de los eventos de salud ocurridos en los doce meses anteriores, tales como distocias, vacas caídas (hipocalcemia) y mortalidad(7).

\section{Evaluación}

De acuerdo al protocolo, las medidas permiten valorar los criterios y con base a ellos, se juzgan cuatro principios, y si cumplen las siguientes condiciones, permite clasificar el nivel de bienestar animal en la unidad de producción:

a) Cuando el valor de todos los principios es mayor a 55 y cuando al menos dos de ellos son mayores a 80 se designa como "excelente" (el bienestar de los animales es del más alto nivel); b) cuando el valor de todos los principios es mayor a 20 y cuando al menos el valor de dos de ellos es mayor a 55 se considera como "buena"; c) cuando el valor de todos los principios es mayor a $10 \mathrm{y}$ cuando al menos el valor de dos de ellos es mayor a 20, se califica como "aceptable" (el bienestar cumple con los requisitos mínimos o ligeramente por encima de estos); d) si no se cumplen las condiciones anteriores, se considera como "no clasificada" (el bienestar es bajo y se considera inaceptable).

La calificación asignada al principio "buena alimentación" se otorga con base a los valores obtenidos en los criterios 1 y 2; la calificación para el principio "buen alojamiento" es otorgado por los valores de los criterios 3 y 5; la calificación para el principio "buena salud" se otorga por los valores de los criterios 6, 7 y 8 y finalmente la calificación para el principio "comportamiento adecuado" se otorga por los valores de los criterios 9, 10, 11 y 12.

Para el cálculo de las puntuaciones de criterio, se aplican tres algoritmos diferentes: a) árbol de decisiones, para comprobar un criterio a nivel de unidad de producción; b) suma ponderada y funciones I-spline ${ }^{(21,22)}$ cuando un criterio está marcado por una sola medida obtenida a nivel individual; esta escala generalmente representa la gravedad de un problema y la proporción de animales que lo tienen (por ejemplo, cojeras leves y graves); c) el uso de los umbrales de alarma

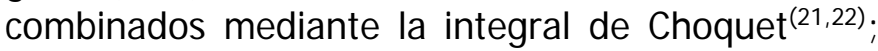
cuando las medidas utilizadas para asignar un valor a un criterio es expresada en diferente escala, los datos se comparan con un umbral de alarma que representa el límite entre lo que se considera anormal y lo que se considera normal, luego se utiliza el número de alarmas como el valor de la medida (Figura 1), los resultados obtenidos se corroboraron con el simulador de $W Q^{\circledR}$ en www1. clermont. inra.fr/wq/index?idsimul\&new=1. 
Figura 1. Diagrama de evaluación multicriterio adaptado de Welfare Quality ${ }^{7)}$

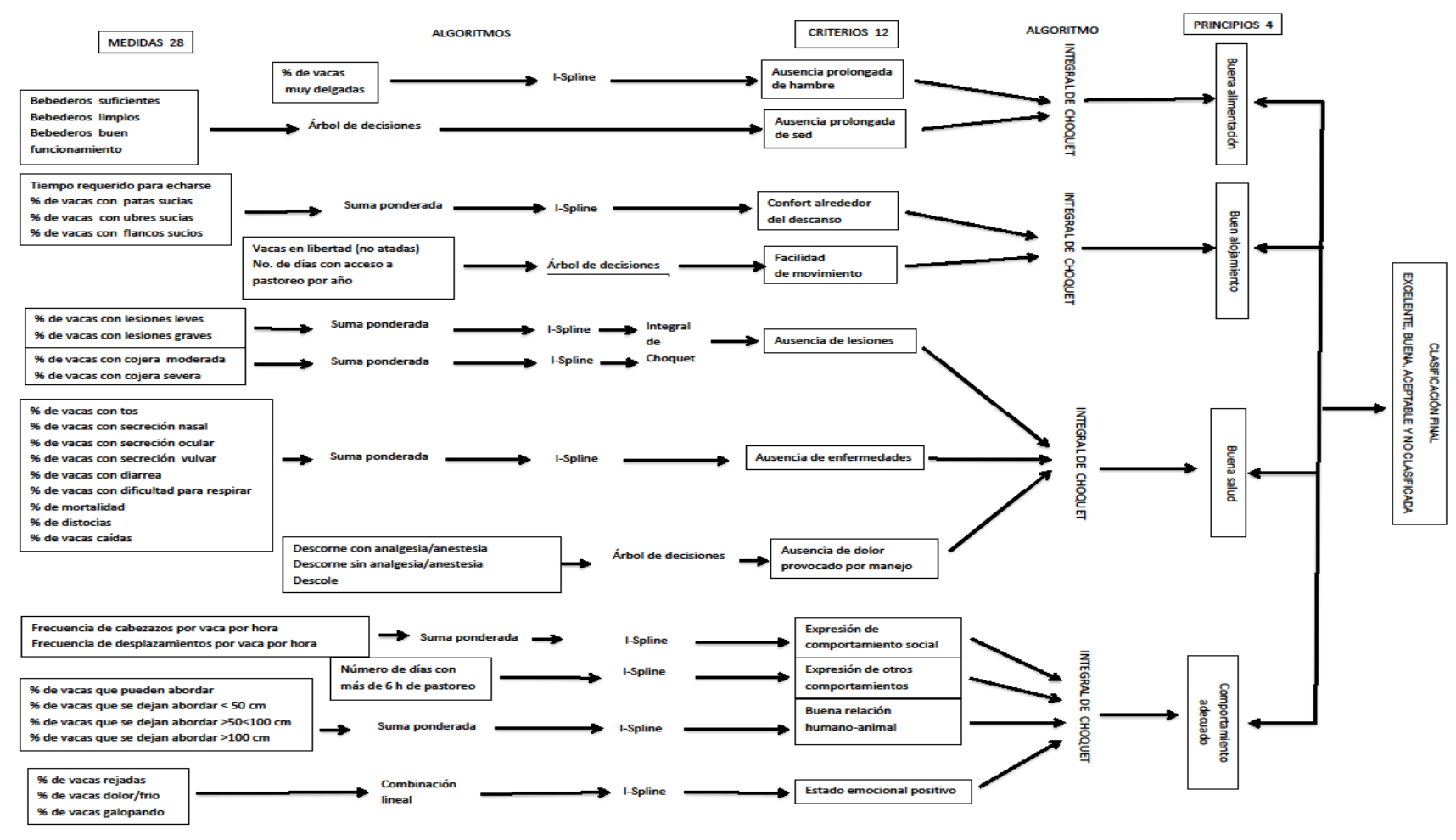

\section{RESULTADOS Y DISCUSIÓN}

\section{Unidades de producción}

Las unidades de producción lechera en los municipios de Tulancingo, Metepec, Acatlán y Cuautepec, del estado de Hidalgo en México, se caracterizan por el manejo con mano de obra familiar, con un promedio de 23 vacas tipo Holstein, en su mayoría con ordeño manual (48 UP) y sólo 4 unidades con ordeño mecánico. La producción promedio fue de 16 L/día destinada al autoconsumo y procesos de quesería locales.

En 22 UP se estimó que las vacas pastorean por más de $6 \mathrm{~h}$, mientras que en las 30 UP restantes, sus vacas pastorean menos de $6 \mathrm{~h}$. La actividad de descanso de las vacas se realiza dentro de las horas de pastoreo, sin contar con un espacio específico para esta actividad.

\section{Evaluación}

En este estudio se evaluaron 29 medidas a nivel individual o de grupo, de éstas, 21 a partir del animal
(Cuadro 1), una a partir de los recursos y siete a través de entrevista utilizando un cuestionario estructurado (Cuadro 2); con estas medidas se obtuvieron los valores de 12 criterios y 4 principios (Cuadro 3); la calificación o puntaje de estos últimos brinda un panorama global de los principales problemas que deterioran el bienestar animal; con los resultados obtenidos el productor tiene la información para priorizar las medidas correctivas que estén a su alcance a fin de mejorar el grado de bienestar.

\section{Principio 1: Alimentación adecuada}

De acuerdo a la clasificación del protocolo $W Q^{\circledR}$ para la condición corporal, el $80.1 \%$ de las vacas tuvieron una condición corporal normal y el $19.3 \%$ restante fueron ubicadas en la categoría de "muy flacas", un valor menor a lo reportado en Argelia(11) bajo condiciones similares a este estudio (33.1\%); sin embargo, en condiciones de estabulación en Rumanía, con vacas tipo Holstein tanto atadas como libres ${ }^{(13)}$ reportaron valores menores (13.2 y $10.2 \%$, 
Cuadro 1. Porcentajes obtenidos en las medidas evaluadas en el animal para 52 unidades de producción aplicando el protocolo Welfare Quality

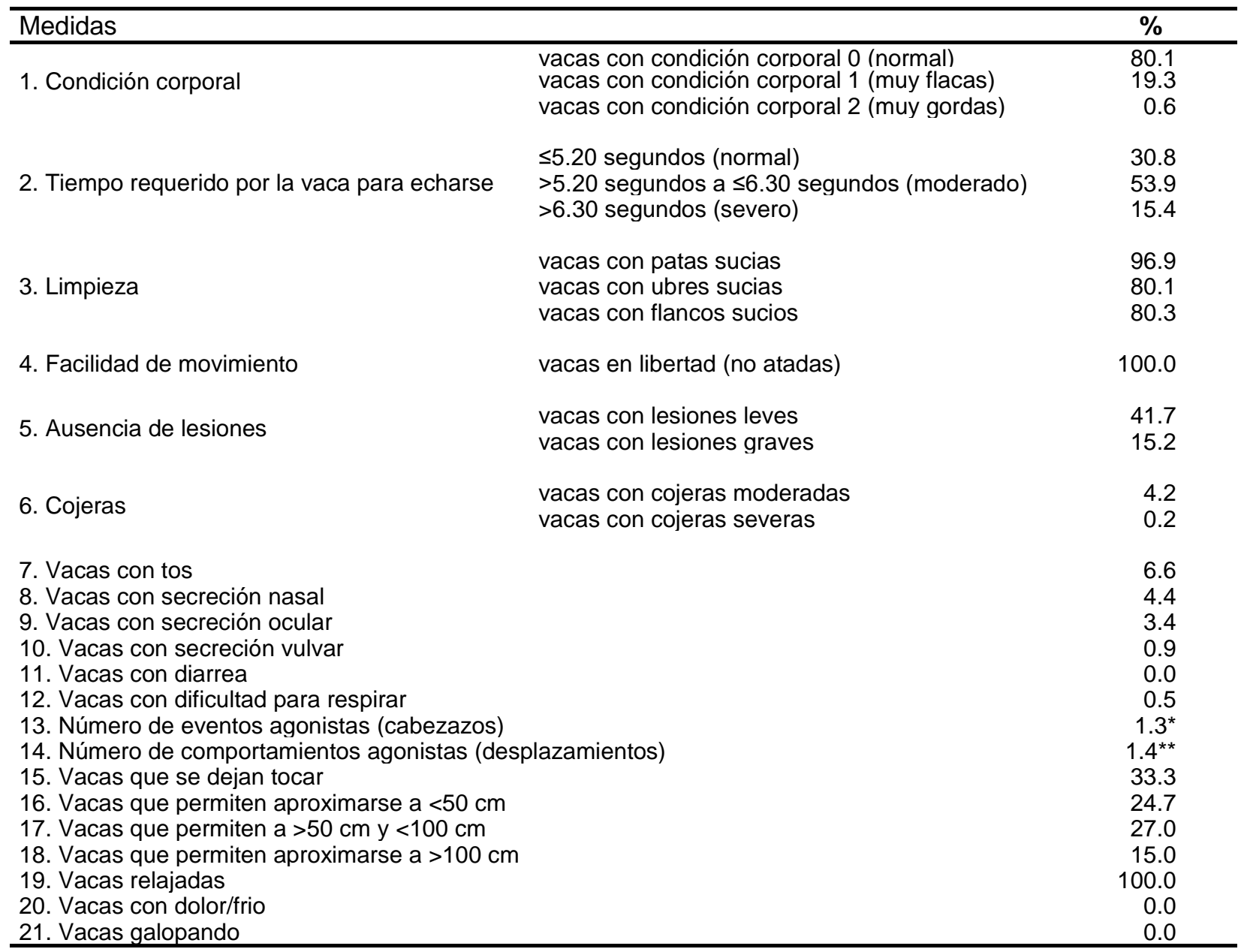

* Expresado en número de cabezazos, este se multiplica por una constante aportada por el protocolo (1.6) a fin de obtener el total de eventos agonistas por vaca por hora.

** Expresado en número de desplazamientos, éste se multiplica por una constante aportada por el protocolo (3.4) a fin de obtener el total de eventos agonistas por vaca por hora.

respectivamente), y en Brasil $^{(9)}$ indican que vacas híbridas en pastoreo, el $7.5 \%$ fueron flacas; este valor de medida está relacionado con la producción, inmunidad ${ }^{(23,24)}$ y cojeras $^{(25)}$; y se ve influenciado también por las características genéticas actuales de las vacas lecheras ${ }^{(26-28)}$. Con los valores obtenidos para condición corporal, la puntuación para el criterio 1 (ausencia prolongada de hambre) en esta investigación fue de 35 puntos. A partir de las medidas de funcionamiento, limpieza y disponibilidad de bebedero/vaca, se obtuvo una puntuación de 54 para el criterio 2 (ausencia prolongada de sed). Con el valor de ambos criterios el principio 1 se ubica con 39 puntos (en una escala de 0 a 100).
Cuadro 2. Valores porcentuales de las medidas a partir de entrevistas con cuestionario estructurado para 52 UP aplicando el protocolo Welfare Quality

\begin{tabular}{lc}
\hline Medidas & $\%$ \\
\hline Mortalidad & 4.7 \\
Distocias & 14.9 \\
Vacas caídas (hipocalcemia) & 3.7 \\
Descorne con analgesia/anestesia & 2.0 \\
Descorne sin anestesia/analgesia & 98.0 \\
Descole & 0.0 \\
Hatos con $<6$ h de pastoreo al día & 42.3 \\
Hatos con $>6$ h de pastoreo al día & 57.7 \\
\hline
\end{tabular}


Cuadro 3. Evaluación para principios y criterios de 52 unidades de producción aplicando el protocolo $W Q^{\circledR}$

\begin{tabular}{ccc} 
& & Puntaje \\
\hline PRINCIPIO 1 & ALIMENTACIÓN ADECUADA & 39 \\
Criterio 1 & Ausencia de hambre prolongada & 35 \\
Criterio 2 & Ausencia de sed prolongada & 54 \\
PRINCIPIO 2 & ALOJAMIENTO ADECUADO & 48 \\
Criterio 3 & Confort en relación al descanso & 41 \\
Criterio 5 & Facilidad de movimiento & 100 \\
PRINCIPIO 3 & SALUD ADECUADA & 23 \\
Criterio 6 & Ausencia de lesiones & 58 \\
Criterio 7 & Ausencia de enfermedad & 45 \\
Criterio 8 & Ausencia de dolor provocado por manejo & 2 \\
PRINCIPIO 4 & COMPORTAMIENTO ADECUADO & 28 \\
Criterio 9 & Expresión de comportamiento social adecuado & 94 \\
Criterio 10 & Expresión de otras conductas & 37 \\
Criterio 11 & Relación humano-animal & 40 \\
Criterio 12 & Estado emocional positivo & 31 \\
\hline
\end{tabular}

\section{Principio 2: Alojamiento adecuado}

Las instalaciones en la actividad lechera son de gran importancia para la producción, la salud y el bienestar de las vacas, debido al efecto directo que tiene en el descanso y determinar el tiempo que la vaca pasa acostada y de pie las $24 \mathrm{~h}^{(29)}$; la disminución en el descanso representa un factor de riesgo en la salud ${ }^{(30,31)}$. De los cuatro principios evaluados, el de alojamiento adecuado fue el que obtuvo mayor puntaje (48), apoyado en mayor medida por el criterio 5 (libertad de movimiento) con el máximo puntaje otorgado. Como resultado del muestreo inicial, se corroboró que en los sistemas de producción familiar no existen cubículos de descanso individual para el ganado, por lo que las medidas de porcentaje de vacas que quedan fuera (total o parcial) de la instalación de descanso, y el porcentaje de vacas que colisionan con este equipo no se realizó. Para evaluar el criterio 3 , un valor que destaca en forma negativa en éste, es la limpieza corporal, donde la suciedad en patas, ubres y flancos rebasan el $80 \%$; el protocolo considera valores normales porcentajes menores a 10 y serios los porcentajes mayores a 19; el resultado de esta medida es grave, ya que se considera un factor de riesgo para mastitis, cojeras y problemas gastrointestinales ${ }^{(18,30)}$, situación no compartida por el productor, que la considera como normal. No se incluyó el criterio 4 (confort térmico) porque de acuerdo al protocolo, este criterio no se evalúa en vacas lecheras.

\section{Principio 3: Salud adecuada}

Este principio es el peor evaluado, obteniendo 23 puntos, debido principalmente al valor más bajo alcanzado en el criterio 8 (dolor provocado por manejo) con solo 2 puntos, dada la ausencia de uso de anestesia/analgesia en el descorne; en contraparte el criterio 6 (ausencia de lesiones) fue el de mayor puntaje obteniendo, 58 puntos, principalmente por la escasa presentación de cojeras graves $(0.16 \%)$. Los datos reportados para hatos pequeños en sistemas similares es de $0.4 \%$ en Chile y $15.6 \%$ Argelia(11,19); algunos sistemas de producción exacerban algunos eventos y disminuyen otros que afectan el $\mathrm{BA}^{(8)}$ como en esta medida. El criterio 7 (ausencia de enfermedad) no rebasa la media de valoración, obteniendo una puntuación de 45; sin embargo habría que reconsiderar el valor, debido a que proviene de un número pequeño de animales y eso eleva el puntaje de la medida, en especial de mortalidad (4.7\%) y distocias ( $14.9 \%$ ) donde el protocolo marca como normal valores inferiores a $2.5 \%$ en ambas medidas.

\section{Principio 4: Comportamiento adecuado}

Para este principio se obtuvo una calificación de 28 puntos; es probable que este resultado sea porque en este estudio, solo se incluyeron cuatro indicadores de valoración objetiva, que hacen referencia a la posición de la cola (relajada, dolor/frío, explorando/alerta y galopando). El mayor 
porcentaje se observó en el indicador "cola relajada". El protocolo de $W Q^{\circledR}$, incluye la propuesta de Wemelsfelder(20), que propone evaluar 20 indicadores de estado emocional, la mayoría de ellos de carácter subjetivo, que hace compleja su aplicabilidad.

La relación humano-animal indicada por la distancia a la que se dejan aproximar las vacas llamada zona de fuga, es el espacio que el animal considera como propio y le da seguridad, e indica el grado de maltrato que pudo sufrir; para esta medida los resultados obtenidos muestran que el $15 \%$ de las vacas evaluadas permitieron una cercanía menor a $1 \mathrm{~m}$; este valor es similar al reportado para ganado en condiciones europeas con un rango de 0.7 a 3.2 $\mathrm{m}^{(32)}$ y $18 \%$ para hatos chilenos en condiciones de semipastoreo ${ }^{(10)}$.

El promedio en los cuatro principios deja claro el incipiente estado de bienestar para la población estudiada, enmarcada en el sistema de producción a pequeña escala (Figura 2).

\section{Clasificación final}

De las UP evaluadas se obtuvieron 0 clasificadas como Excelente, 0 clasificadas como Buena, 52 clasificadas como Aceptable y 0 No clasificadas. El total de las UP oscilan en condiciones mínimas de bienestar.

Los resultados obtenidos en el presente estudio son similares a los obtenidos en el simulador de $W Q^{\circledR}$, alimentado con los datos a partir de principios;

Figura 2. Valores promedio para Principios de 52 UP usando el protocolo Welfare Quality ${ }^{\circledR}$

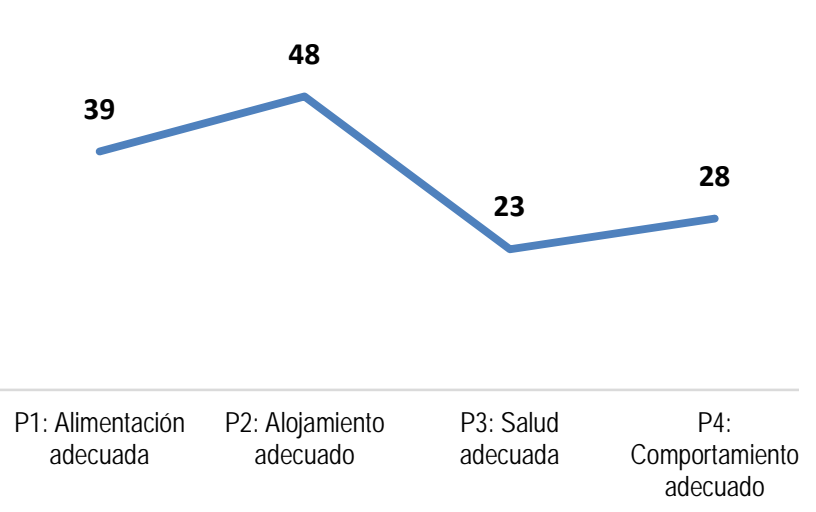

cuando se utiliza la información a partir de medidas, no arroja evaluación; es probable que sea por la diferencias naturales entre el sistema de producción de origen y el sistema a pequeña escala o familiar como el de este estudio.

\section{CONCLUSIONES E IMPLICACIONES}

Las vacas lecheras de la población evaluada en el sistema de producción a pequeña escala se encuentran en condiciones mínimas de bienestar; el uso del protocolo de evaluación de BA Welfare Quality ${ }^{\circledR}$ es una herramienta útil para valorar ese bienestar. La aplicabilidad del protocolo en algunos aspectos no es total y se hace necesario el uso de indicadores más acordes al sistema de producción familiar, como los sombreaderos, que pueden contribuir a disminuir algún sesgo, al no poder incluir la medida de vacas fuera o parcialmente fuera del área de descanso en el Principio 2 (alojamiento adecuado), dado que no existe en este sistema de producción; para ello, se tendrán que validar y probar. Además, es probable que al incluir la medida de distancia a la que pastorean, pueda disminuir el sesgo de la medida atada o no atada, en el Principio 2, criterio 5 (facilidad de movimiento).

\section{AGRADECIMIENTOS}

A la Fundación Hidalgo Produce A. C. que financió el presente estudio. Al Centro Universitario UAEM Amecameca. Al Programa en Ciencias Agropecuarias y Recursos Naturales (PCARN). Al Instituto de Ciencias Agropecuarias (ICAP) de la UAEH. A los productores por su participación voluntaria.

\section{LITERATURA CITADA}

1. Blokhuis HJ, Keeling LJ, Gavinelli A, Serratosa J. Animal welfare's impact on the food chain. Trends Food Sci Tech 2008; 19:S79-S87.

2. Knierim $U$, Winckler $C$. On-farm welfare assessment in cattle: validity, reliability and feasibility issues and future perspectives with special regard to the Welfare Quality ${ }^{\circledR}$ approach. Anim Welfare 2009; 18(4):451-458.

3. Canali E, Keeling L. Welfare Quality ${ }^{\circledR}$ project: from scientific research to on farm assessment of animal welfare. Ital J Anim Sci 2010;8(2s):900-903. 
Miguel Ángel Silva Salas, et al. / Rev Mex Cienc Pecu 2017;8(1):53-60

4. Webster J. The assessment and implementation of animal welfare: theory into practice. Rev Sci Tech OIE 2005; 24(2): 723.

5. Des Roches A, et al. The major welfare problems of dairy cows in French commercial farms: an epidemiological approach. Anim Welfare 2014;23(4):467-478.

6. OIE. World Organization for Animal Health. Terrestrial Animal Health Code. Twenty-first ed. Roma. 2012.

7. $W^{\circledR}$. Welfare Quality Consortium. Welfare quality assessment protocol for cattle. Netherlans. 2009.

8. Coignard M, Guatteo R, Veissier I, des Roches A, Mounier L, Lehébel A, Bareille N. Description and factors of variation of the overall health score in French dairy cattle herds using the Welfare Quality ${ }^{\circledR}$ assessment protocol. Prev Vet Med 2013;112(3):296-308.

9. Franchi GA, Garcia PR, da Silva IJ O. Welfare quality applied to the Brazilian dairy cattle. J Anim Behav Biometerol 2014;2(2):60-65.

10. Arraño CBA, Flor E. Preliminary study of the use of protocol to assessment the welfare of dairy cattle using animal-based observations. Arch Med Vet 2007;30(3):239-245.

11. Benatallah A, Ghozlane F, Marie M. Dairy cow welfare assessment on Algerian farms. Afr J Agric Res 2015;10(9):895-901.

12. Stanković B, Hristov S, Ostojić-Andrić D, Zlatanović Z, Samolovac $\mathrm{L}$, Maksimović $\mathrm{N}$. The most common health disorders and welfare of dairy cows and calves. Biotechnol Anim Husb 2014;30(4):549560.

13. Popescu S, Borda C, Diugan EA, Spinu M, Groza IS, Sandru CD. Dairy cows welfare quality in tie-stall housing system with or without access to exercise. Acta Vet Scan 2013;55(1):43.

14. SIAP-SAGARPA. Servicio de Información Agroalimentaria y Pesquera-Secretaría de Agricultura Ganadería Desarrollo Rural Pesca y Alimentación. Producción de leche de bovino en México. México. 2012.

15. Arriaga J C, Heredia ND. Importancia de los sistemas de producción de leche a pequeña escala en México. Congreso nacional de producción, comercialización y nutrición de la leche y derivados. México: Editor UAEMex; 2013.

16. Edmonson AJ, Lean IJ, Weaver LD, Farver T. A body condition scoring chart for Holstein dairy cows. J Dairy Sci 1989; 72(1):68-78.

17. Hughes J. A system for assessing cow cleanliness. In Practice 2001;23(9):517-524.

18. Welfare Quality Consortium. Welfare Quality Reports No. 11 Netherlans. 2009.

19. Tadich N, Flor E, Green L. Associations between hoof lesions and locomotion score in 1098 unsound dairy cows. Vet J 2010; 184(1):60-65.
20. Wemelsfelder F, Lawrence AB. Qualitative assessment of animal behaviour as an on-farm welfare-monitoring tool. Acta Agri Scan AAN 2001;51(S30):21-25.

21. Landa GE. Puesta a punto del método Welfare Quality ${ }^{\circledR}$ para valorar el bienestar animal de las granjas del grupo Lacturale [tesis licenciatura]. Navarra España: Universidad Pública de Navarra; 2011.

22. Urreaga OI. Valoración del bienestar animal en la granja de vacuno de leche SAT Etxeberri según Welfare Quality ${ }^{\circledR}$ [tesis licenciatura]. Navarra España: Universidad Pública de Navarra; 2013.

23. Sumner JM, McNamara JP. Expression of lipolytic genes in the adipose tissue of pregnant and lactating Holstein dairy cattle. J Dairy Sci 2007; 90:5237-5246.

24. Roche JR, Friggens NC, Kay J K, Fisher MW, Stafford KJ, Berry DP. Invited review: Body condition score and its association with dairy cow productivity, health, and welfare. J Dairy Sci 2009; 92(12):5769-5801.

25. Randall L, Chagunda MJ, Mason MGG, Archer C, Green SC, Huxley LE. Low body condition predisposes cattle to lameness: An 8-year study of one dairy herd. J Dairy Sci 2015;98(6):3766-3777.

26. Lucy MC, Verkerk GA, Whyte BE, Macdonald KA, Burton L, Cursons JRRRT, Holmes C W. Somatotropic axis components and nutrient partitioning in genetically diverse dairy cows managed under different feed allowances in a pasture. J Dairy Sci 2009;92:526539.

27. Rodríguez-Martínez H, Bergsten C, Lidfors L, Gunnarsson S, Algers B, Emanuelson U. La eficiencia reproductiva en vacas lecheras de alta producción: Es sostenible con las prácticas de manejo actuales. IVIS Int Vet I nform Serv eds, Reviews in Vet Med. Ithaca NY, USA. 2008.

28. Matthews L, Cameron C, Sheahan A, Kolver E, Roche J. Associations among dairy cow body condition and welfare-associated behavioral traits. J Dairy Sci 2012;95(5):2595-2601.

29. Margerison J, Lau J, Hedley M, Horne D, Hanly J, Powell N, Shilton A. Lying and feeding behaviour of dairy cows at pasture and housed in free stalls fitted with sand and water filled matts. Nutrient management for the farm, catchment and community. Currie LD, Christensen CL editors. Occasional Report. 2014;27.

30. De Vries M, Bokkersa EAM, van Reenenb CG, Engelc B, van Schaikd G, Dijkstrad T, de Boer IJM. Housing and management factors associated with indicators of dairy cattle welfare. Prev Vet Med 2015; 118:80-92.

31. Ito KVK, LeBlanc MAG, Weary SJ . Lying behavior as an indicator of lameness in dairy cows. J Dairy Sci 2010;93(8):3553-3560.

32. Whay $H$, Main $D$, Green $L$, Webster A. Observations and investigation of farm. Vet Rec 2003; 153:197-202. 